\title{
Rol investigador en la identidad del profesorado de Educación física y Psicología educativa: estudio comparativo Investigative role in the identity of teachers of Physical Education and Educational Psychology: comparative study
}

\author{
*Tahimi Achilie-Valencia, **Arturo Rodriguez-Zambrano, **Jhonny Villafuerte-Holguín \\ *Pontificia Universidad Católica del Ecuador Sede Esmeraldas (Ecuador), ${ }^{* *}$ Universidad Laica Eloy Alfaro de Manabí (Ecuador)
}

\begin{abstract}
Resumen. El estudio de la identidad del profesorado sigue siendo un tema de relevancia que se vincula a los cambios en la política pública, pero también a su salud física y emocional. Este trabajo tiene como objetivo comparar los cambios en la identidad del profesorado que asumió el rol investigativo en los campos del conocimiento de Educación Física y Psicología Educativa. Desde el paradigma socio crítico se utiliza el enfoque mixto de la investigación. Los participantes son treinta y cinco (35) profesores afiliados a universidades públicas y privadas localizadas en las provincias de Esmeralda y Manabí en Ecuador. Se ajustó y aplicó el cuestionario para la Construcción de la Identidad Docente de los Profesionales de la salud propuesta por Jara y Mayor-Ruiz (2018). Se administró un cuestionario tipo Likert, 12 entrevistas a profundidad y 5 grupos focales (7 personas por grupo focal) durante el periodo 2019-2020. Entre los resultados se presenta el análisis de las voces de los participantes que evidencia cambios en las motivaciones para la enseñanza y la participación en procesos de investigación científica a nivel local y regional. El análisis estadístico permitió determinar la situación de los participantes respecto a las dimensiones: Formación investigativa, reflexión del proceso educativo, desempeño profesional, y elementos afectivos de la identidad. Se concluye que todos los participantes han fortalecido el rol docente sin importar el campo de conocimiento al que pertenecen.
\end{abstract}

Palabras clave: Actividad física, educación superior; formación doctoral; identidad; profesorado.

\begin{abstract}
The study of teachers' identity is a relevant issue linked to changes in public policy and physical and emotional health. This work aims to compare the changes in the identity of the teaching staff who assumed the investigative role in the fields of knowledge of Physical Education and Educational Psychology. From the socio-critical paradigm. It used the mixed research approach. The participants are thirty-five (35) professors affiliated with public and private universities in Esmeralda and Manabí in Ecuador. The Construction of the Teaching Identity of Health Professionals questionnaire proposed by Jara and Mayor-Ruiz (2018) was adjusted and applied. It administrated a Likert-type questionnaire, 12 in-depth interviews, and five focus groups (7 people per focus group) during the 2019-2020 period. Among the results, the analysis of the participants' voices is presented, which shows changes in the motivations for teaching and participation in scientific research processes at the local and regional levels. The statistical analysis determined the participants' situation regarding the dimensions: Investigative training, the reflection of the educational process, professional performance, and affective elements of identity. It concluded that all participants had strengthened the teaching role regardless of their field of knowledge.
\end{abstract}

Keywords: Physical activity, higher education; doctoral training; identity; faculty.

\section{Introducción}

El estudio de la identidad del profesorado sigue vigente a nivel global debido a que su evolución se relaciona con los cambios en las políticas públicas y con los procesos formativos que ellos asumen.

El trabajo investigativo en la línea identidad del profesorado se ha incrementado en las últimas décadas, utilizando el enfoque de la dialógica (Uitto, Kaunisto, Syrjälä, \& Estola, 2015). Las investigaciones involucran

Fecha recepción: 29-03-21. Fecha de aceptación: 16-06-21

Jhonny Villafuerte

jhonny.villafuerte@gmail.com el papel relevante de la formación inicial, la interpretación de las reformas en los sistemas educativos y los procesos de perfeccionamiento profesional que se asumen (Tapia, Granados, \& Fernández, 2017). Así, la formación doctoral consolida los conocimientos y habilidades de la profesión (Jara \& Mayor-Ruíz, 2018), lo que apoya al profesorado para el desempeño de las funciones de docencia, investigación y vinculación con la sociedad (Morales \& Rueda, 2019).

Entre los antecedentes del proceso de evaluación de la calidad de las universidades y escuelas politécnicas de Ecuador, se señala la Ley de Educación Superior del año 2000 y la Resolución expedida en diciembre del 2002 por el Concejo Nacional de Educación Superior 
[CONESUP]. Así, la asignación de fondos del estado ecuatoriano dependía de las transferencias que las Instituciones de Educación Superior (IES) habían recibido en el año anterior. Por lo tanto, no eran considerados los factores tales como el cumplimiento de metas científicas, los objetivos de las políticas públicas, ni los aportes al desarrollo social. En dicho escenario, los líderes de las universidades fueron en muchos casos perennizados, situación que facilitó acciones indebidas y como resultado, la educación superior de Ecuador fue influenciada por procesos de mercantilización que imponía dinámicas de exclusión y abuso (Ramírez, CevallosVallejos, Díaz-Reinoso, Grijalva, Rojas et al., 2016).

Las universidades estatales fueron tildadas como de mala calidad para destacar las bondades de la educación privada, pero el ingreso a las universidades elitistas llegó a depender del poder adquisitivo e influencias de los aspirantes y de sus familias. Así, los procesos de desarrollo productivo se limitaron a la exportación de commodities carentes de valor agregado, mientras que el país importaba bienes industrializados y tecnológicos (Ramírez et al., 2016). El modelo de evaluación utilizado por CEAACES en el 2015 siguió los principios de la excelencia académica e incluye los criterios (a) Organización, (b) Academia, (c) Investigación, (d) Vinculación con la sociedad, (e) Recursos e infraestructura y (f) Estudiantes (Moreira, Fleitas, Veliz, \& Vinces, 2016). Se trata de un sistema que busca de forma constante y sistemática la pertinencia, producción óptima, transmisión del conocimiento y desarrollo del pensamiento (Cevallos, 2018). No obstante, no considera los procesos puestos en marcha, sino que se centra en el análisis de los resultados (Véliz-Briones, 2018).

En este punto, los autores declaran que el 'rol investigador del profesorado' se refiere a la participación en procesos que generan nuevos conocimientos que aportan al desarrollo de las naciones (Darley \& Luethge, 2016). Se agrega que el profesorado se debe mantener en actividad investigativa para contribuir a la economía del conocimiento, ser relevantes para el contexto y garantizar que los estudiantes se eduquen mediante el uso de la investigación empírica y teórica. Por su parte, Keiler (2018), argumenta que el rol investigador del profesorado potencia en el alumnado la comprensión profunda del contenido y desarrolla las habilidades para el uso del método científico. Por su parte, Vallejo-López (2020) argumenta que el rol investigador del profesorado influye positivamente en el desarrollo académico de las instituciones educativas y propicia que el alumnado asuma el compromiso social y moral para ejecutar los proyectos de investigación a lo largo de la carrera y en el futuro ejercicio profesional.

La educación superior de Ecuador continúa la ejecución de procesos de mejoramiento mediante indicadores sobre la internacionalidad de las universidades y la cooperación mediante redes de investigación (GuerreoAray, Suástegui-Solórzano, \& Zambrano-Vera, 2019). Se trata de cambios necesarios en la educación superior en América Latina que además se vinculan con la infraestructura y la calidad de la enseñanza (Bernate, Guataquira Romero, Romero-Melo, \& Reyes-Escobar, 2020). Por lo tanto, se confirma que el concepto de calidad en la educación superior tiene características de pluridimensionalidad, que contrasta todas las funciones sustantivas y procesos universitarios con la satisfacción de las partes interesadas (Orozco-Inca, Jaya-Escobar, Ramos Azcuy, \& Guerra-Bretaña, 2020).

Este trabajo se ha planteado a partir de la pregunta: ¿cuáles son los cambios en la identidad y en el rol investigativo del profesorado de Esmeraldas y Manabí de Ecuador?

La hipótesis nula planteada es: No existen diferencias significativas entre los campos del conocimiento y el rol investigativo del profesorado de Educación Física y Psicología Educativa antes y después de la formación investigativa doctoral.

Este trabajo tiene como objetivo comparar los cambios en la identidad del profesorado que asumió el rol investigativo en los campos del conocimiento de Educación Física y Psicología Educativa.

\section{La identidad del profesorado}

El rol de los investigadores se relaciona con la identificación de problemas en niveles macro, meso y micro, así como con el desarrollo de soluciones (Antuña, 2014). Entre los retos más relevantes del profesorado está el armonizar el rol profesional con los otros roles a ejercer en la sociedad (Antuña, 2014). El profesorado adopta diversos roles en la práctica profesional, tales como ser tutores de procesos de titulación, directivos de los espacios de difusión de la ciencia o el liderazgo de proyectos de investigación (Fastuca \& Wainerman, 2015). Desde esta línea, es posible sostener que la identidad del profesorado no es algo que se obliga, más bien se negocia y fortalece a lo largo de la vida (Fastuca \& Wainerman, 2015). A estas perspectivas comunes, se le suman los cambios en las estructuras sociales que, en la actualidad, se relacionan con aspectos como la inclusión, la diversidad y el género (Morales, 2015).

El constructo identidad del profesorado está sujeto a 
constantes transformaciones que se producen debido a los cambios en la academia, los desafíos de los procesos formativos, y las prácticas y anhelos personales (Alonso, Lobato \& Arandia, 2015; Guzmán, 2017).

La identidad de las personas investigadoras se relaciona con la formación doctoral, siendo este el escenario donde se consolidan los conocimientos y habilidades de la profesión académica (Jara \& Mayor-Ruíz, 2018). Esta construcción es individual y dinámica la que se ve influenciada por los diversos procesos de socialización y los elementos sociohistóricos acontecidos en el recorrido profesional de las personas que investigan. Desde esta línea, es posible sostener que la identidad del profesorado es algo que se negocia y fortalece a lo largo de la vida. Esta construcción es individual y dinámica y se ve influenciada por el contexto sociohistórico (GajardoAsbún, 2019).

Se trata de responsabilidades que requieren de asignaciones presupuestarias y de la gestión del talento humano en un eco ambiente cargado de altas exigencias (Morales \& Rueda, 2019). Su desempeño profesional suele estar medido por instrumentos institucionales (Jiménez, 2019) y el cumplimiento de metas altamente exigentes (Bermejo et al., 2020).

\section{La función investigativa del profesorado}

Las funciones sustantivas de la universidad involucran la docencia, la investigación y la vinculación social. A su vez, estas funciones promueven el planteamiento de cambios dentro de las propias instituciones por lo que se vuelven cada vez más pertinentes para el desarrollo institucional y del contexto (Pacheco, 2014). En este sentido, la función investigativa articula la ejecución de actividades de recolección de información y datos que son contrastados con la revisión bibliográfica para el estudio de los hechos relevantes acontecidos en el territorio donde se desempeñan (Padilla, López \& Rodríguez, 2015).

De las funciones sustantivas universitarias emergen sentimientos que ponen a prueba la identidad del profesorado desde su esencia humana (Granados, Tapia, \& Fernández, 2017). Por lo tanto, elementos tales como la motivación al autoaprendizaje, el compromiso, la responsabilidad y la reflexión son parte de sus prácticas cotidianas (Bell, 2017), las que permiten mejorar su desempeño como profesores y como investigadores para proponer mejoras en la ejecución de las funciones de docencia, vinculación con la sociedad y sobre todo en la investigación científica (Morales \& Rueda, 2019).

En tal escenario, el profesorado se encuentra ante la necesidad de revisar permanentemente su identidad en consonancia con sus decisiones de vida y los cambios previstos y no previstos en su contexto (Rueda, Acosta, \& Cueva, 2020), donde la institucionalidad universitaria da legitimidad a las actividades investigativas, reconociéndolas como importantes para la sociedad.

Finalmente, las universidades innovan sus modelos educativos con la intención de responder a las expectativas y necesidades del medio circundante (Sáez, Gil, \& Martínez, 2021), pero se trata de un proceso arduo, serio y a largo plazo que es puesto en marcha desde la función investigativa.

\section{La formación investigativa a nivel doctoral}

Los programas de formación doctoral se relacionan con la generación de conocimiento a través de la elaboración de tesis y otros productos científicos avanzados que aportan a la adquisición de las habilidades (1) investigativas, (2) reflexivas, (3) de resolución de problemas, (4) comunicativas y (5) metodológicas» (Ismail \& Meerah, 2012, p. 245). Tales competencias forman parte esencial de la identidad del profesorado donde las culturas académicas, estrechamente ligadas a la diversidad humana permiten el encuentro y la cohesión entre los significados y las posturas de quienes lideran la investigación en las universidades Iberoamericanas (Palomar, 2013).

Los aportes de la investigación científica permiten examinar las políticas públicas dirigidas al desarrollo de la sociedad (Palamidessi, Gorostiaga, \& Suasnábar, 2014). Para su puesta en marcha se elaboran modelos curriculares de las instituciones de educación superior (Izquierdo \& Loarte, 2014), y se construyen marcos normativos generales y específicos para cada campo del conocimiento (Pacheco, 2014).

Según Fastuca \& Wainerman (2015), las competencias investigativas que se fortalecen en los programas de doctorado se relacionan con el estudio de las teorías, el uso de instrumentos de recolección de datos o de información, y su análisis. También está otro grupo de actividades explícitas que resultan difíciles de trasmitir porque corresponden a realizaciones a las que se llega sin saber cómo hacerlo y que se obtienen solamente con la práctica constante.

Los procesos formativos doctorales deben acoplarse a las demandas del contexto con sus variantes económicas, sociales y culturales, pero en la actualidad cada vez las fronteras dejan de tener como límite los países. Esto implica experticia alrededor de lineamientos teóricos y las orientaciones metodológicas (Cuevas, 2016), con 
la esperanza de que el alumnado sea capaz de aprender a aprender, aprender a ser y aprender a hacer (Rojas, 2016). Su propósito es aportar en la búsqueda de soluciones de las problemáticas que afectan a las comunidades mediante diversos servicios profesionales (Polaino \& Romillo, 2017). Por ello, los doctorandos ejecutan los procesos formativos con ayuda de los tutores (Núñez \& Santesteban, 2017).

La formación de investigadores reafirma su pertinencia y responsabilidad en la gestión del saber. Este proceso a su vez demanda al profesorado, involucrarse en grupos solventes de investigación como una práctica que transforma su identidad profesional (Rivera, Espinosa, \& Valdés, 2017; Saltos, Odriozola, \& Ortiz, 2018), y las universidades se convierten en los escenarios de producción, difusión y utilización del conocimiento (García, Jara, \& Cedillo, 2018).

En este punto, la formación doctoral contribuye con el mejoramiento de la enseñanza en los diferentes dominios (Difabio de Anglat, Portela de Nieto, GelonchVillarino, Muscará \& Boarini de Dutto, 2018), donde la capacidad crítica y de innovación permite identificar y solucionar problemas del conocimiento (Nieva \& Mendoza, 2019). Este aporte se manifiesta en la pertinencia de los modelos educativos que son puestos a prueba en la búsqueda de las soluciones requeridas y armonía con el contexto donde se desempeñan los involucrados (Bermejo, Pulido, Galmés, Serra, Vidal et al., 2020).

Entre los estudios previos revisados se cita el trabajo de Moya \& Alonso (2016) quienes sostienen que las competencias del profesorado universitario se fundamentan en la integración y activación de recursos didácticos, pedagógicos, materiales y humanos. Ellos afirman que el trabajo en el aula universitaria logra la profundización, uso, ajuste, conciliación y adaptación de los contenidos mediante el currículo, y aporta a la construcción del perfil de los profesionales. En este mismo recorrido, Nghia \& Tai (2017) manifiestan que el estudio de la identidad del profesorado en la etapa de formación se centra en la revisión de sus experiencias iniciales. Esta etapa se caracteriza por una alta carga de imaginaciones idealistas o expectativas que resultan naturales al inicio del proceso formativo. Se añade el trabajo de Keiler (2018) quien encontró que cuando los cambios en el entorno de los docentes son radicales, estos pueden afectar su identidad y con ello, incidir sobre los enfoques de la enseñanza que utilizan. Por ello, el profesorado transita por los diversos modelos de enseñanza ya que ellos procuran convertirse en los maestros que siempre habían querido ser. Finalmente, se agrega el trabajo de Anversa, Souza, Both, da Costa, Batista et al. (2020), quienes observaron el desempeño del profesorado y su identidad ante las políticas educativas desde las dimensiones de la representación profesional, las prácticas profesionales y las pasantías de investigación.

\section{Material y método}

Esta investigación acude al paradigma socio crítico a partir de su capacidad para generar mejoras en el desempeño profesional de los individuos (Alvarado y García, 2008). Se acude al enfoque mixto de la investigación para estudiar las transformaciones originadas en el rol investigativo de un grupo de profesores durante el proceso de formación doctoral. El enfoque mixto de la investigación permite un nivel mayor de entendimiento del objeto investigativo, identificando tanto las representaciones individuales como las generalizaciones colectivas (Moscoso, 2017). Para el análisis de la información se considera el modelo de Edwards \& Edwards (2016), en el que se articulan los factores cultura, contextos y experiencias vividas al proceso de construcción de la identidad de los participantes.

\section{Los participantes}

La muestra utilizada es de tipo aleatoria por conglomerado, donde las condiciones mínimas para participar fueron cursar o haber culminado estudios doctorales entre 2012 y 2020, trabajar en universidades localizadas en las provincias de Manabí o Esmeraldas, y aceptar la invitación a tomar parte del estudio.

Se trata de un grupo heterogéneo conformado por treinta y cinco profesores de las áreas de Educación Física y Psicología Educativa. Los participantes fueron mujeres (53\%) y hombres (47\%) con edades comprendidas entre 38 y 51 años (promedio 45,32 años), afiliados a cuatro universidades públicas y privadas, localizadas en las provincias Esmeraldas y Manabí en Ecuador. Los criterios para la selección de los participantes que se administraron fueron: (1) haber iniciado estudios doctorales a partir del año 2012; (2) haber realizado estancias en universidades extranjeras; y (2) haber aceptado voluntariamente la invitación a participar en esta investigación. Véase tabla 1.

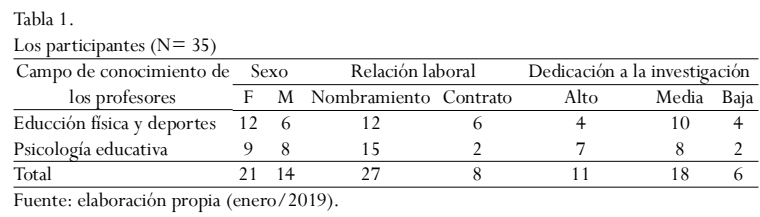




\section{Instrumentos}

Durante la investigación se ejecutaron doce (12) entrevistas a profundidad y se efectuaron cuatro (4) grupos focales con la participación de entre cuatro (4) a cinco (5) personas cada ocasión, debido a la dispersión geográfica de los participantes.

Entrevista a profundidad: La guía de entrevista fue diseñada $a d$. hoc. por el equipo investigador con el objetivo de recoger información respecto a los antecedentes de la identidad de los informantes. Las categorías abordadas son: (1) Las razones que le llevaron a ser docente universitario, (2) La política pública para la educación superior y el trabajo del profesor universitario y (3) Sacrificios hechos por los informantes para realizar los estudios doctorales. El instrumento consta de 8 preguntas que fueron evaluadas por panel de tres especialistas de los campos de la Formación docente, Educación y Psicología. Ellos laboran en la Universidad Católica de Ecuador Sede Esmeraldas. Las recomendaciones del panel de especialistas se relacionaron a la redacción de las preguntas y al incremento de la categoría '(3) Sacrificios hechos por los informantes para realizar los estudios doctorales'. Una vez realizadas las correcciones sugeridas se ejecutó las entrevistas a los informantes de manera presencial en sus lugares de trabajo.

Grupo focal: El propósito de este instrumento fue recoger información de los participantes respecto a los cambios en la identidad respecto al rol investigativo. Véase tabla 2. Los aspectos trabajados fueron (1) Promoción de la ciencia en la formación de profesionales del siglo XXI; (2) Participación de la comunidad científica global; y (3) Gestión de la ciencia para la superación de los problemas locales. El instrumento fue evaluado por panel de especialistas de los campos de la Formación docente, Psicología y Educación Física de la Universidad Laica Eloy Alfaro de Manabí. El instrumento en su versión inicial constaba de 12 preguntas detonantes que fueron reducidas a 6 por recomendación del panel de especialistas.

Cuestionario tipo Likert: Se utilizó el Cuestionario para la Construcción de la Identidad Docente de los Profesionales de la salud de Jara y Mayor-Ruiz (2018). El instrumento fue ajustado a las condiciones sociopolíticas de Ecuador. La versión utilizada consta de 34 ítems distribuidos en las dimensiones (1) formación, (2) reflexión del proceso educativo, (3) desempeño profesional, y (4) elementos afectivos de la identidad. El instrumento fue evaluado por medio del índice Alfa de Cronbach, el que reportó 0.72 , dando cumplimiento a los estándares requeridos para este tipo de estudios
(Domínguez \& Lara, 2015).

\section{Procedimiento}

El protocolo seguido en la ejecución de este estudio es explicado a continuación:

1. Selección de los participantes (enero/2019)

Los participantes fueron contactados previamente por los miembros del equipo investigador según su residencia en las provincias de Manabí o Esmeraldas.

2. Adaptación de cuestionario para la Construcción de la Identidad Docente de los Profesionales (Jara y Mayor-Ruiz, 2018). El equipo investigador realizó la adaptación del cuestionario al leguaje y condiciones contextuales de Ecuador.

3. Validación de instrumento cuestionario Likert.

Se ejecutó una prueba piloto con la colaboración de docentes en formación de la ULEAM, cuyos resultados fueron utilizados para ejecutar las pruebas de confiabilidad de Alfa de Cronbach.

4. Elaboración de instrumentos entrevista y grupos focales

El equipo investigador realizó una revisión de literatura sobre estudios similares para identificar los instrumentos entrevistas y grupos focales que otros investigadores hayan ejecutado. Posteriormente, se elaboró las guías de los dos instrumentos requeridos.

5. Validación de instrumentos por panel de expertos

Los instrumentos guía de entrevista y guía de grupo focal fueron evaluados por panel de especialistas de los campos de la Formación docente, Psicología y Educación Física de la Universidad Laica Eloy Alfaro de Manabí. Los especialistas tuvieron 20 días para la lectura y emisión de observaciones sobre los instrumentos.

6. Ejecución del cuestionario Likert (mayo-junio/ 2020).

Su ejecución se realizó mediante un formulario de Google debido a las regulaciones de distanciamiento social por la epidemia de COVID19.

7. Ejecución de entrevistas (marzo/2019-febrero/ 2020). Los participantes fueron contactados previamente por los miembros del equipo investigador según su residencia en las provincias de Manabí o Esmeraldas. Las entrevistas tomaron en promedio 40 minutos, fueron grabadas y transcritas por la persona que ejecutó las entrevistas. La validación de la información fue hecha por los mismos entrevistados diez días posteriores a la ejecución de las entrevistas. El proceso se ejecutó entre marzo/2019 y febrero/2020.

8. Ejecución de grupos focales (septiembre-octu- 
bre/2020).

Los grupos focales fueron organizados en cuanto a fecha y hora por medio de llamada telefónica. Los grupos focales se realizaron mediante la aplicación informática Zoom, siguiendo las normativas de distanciamiento social vigentes en septiembre/2020 en Ecuador. El análisis de la información colectada se realizó con la colaboración de miembros del proyecto, quienes no participaron en la ejecución de los grupos focales. La información considerada en este estudio es aquella que alcanzó el 90\% de coincidencia en el análisis de triangulación.

9. Triangulación de la información recogida (septiembre/2020-febrero/2021).

Se ejecutó la triangulación de la información obtenida en las entrevistas y grupos focales, los datos obtenidos en el cuestionario Likert y la literatura revisada.

\section{Análisis de resultados}

Para el reconocimiento de las categorías (1) promoción de la ciencia en la formación de profesionales, (2) participación de la comunidad científica global y (3) gestor de la ciencia para la superación de los problemas locales derivadas de los resultados cualitativos se aplicó la técnica de análisis de contenido, con la asistencia del programa Atlas. Ti 8.0.

Por su lado, el análisis cuantitativo de las dimensiones del estudio se realizó a través de sus las funciones no paramétricas asistidas por el software SPSS versión 25.

\section{Resultados y discusión}

Los resultados de esta investigación son expuestos a continuación.

\section{La identidad del profesorado de Esmeraldas y}

\section{Manabí de Ecuador}

Del análisis de las voces de los participantes se indica que la motivación intrínseca es quizás la mayor fuerza que les ha movido a convertirse en profesores. Así, el deseo de procurar la superación y perfeccionamiento profesional ha permitido asumieran el reto que representa la formación doctoral en el extranjero.

Los participantes han expresado haber realizado sacrificios personales, familiares, entre otros, para responder responsablemente a las políticas públicas de la educación superior de Ecuador. De las entrevistas de los participantes se extrae:

E1.01: «Sobre las razones de elegir ser profesor, pienso que fue la vocación y la pasión por la enseñanza de educación física. Mediante esta profesión aporto a la salud y calidad de vida de las personas».

E4.03: Siempre me ha gustado enseñar, he estado identificada con esta labor desde que era muy joven. Así aporto a la economía de mi país.»

E2.04: «Las cosas cambiaron con las reformas en la política para la educación superior. Mi motivación para ser profesor tiene ese origen.»

E4.07: «La formación doctoral me ha demandado alta dedicación a procesos investigativos. He realizado sacrificios que me alejan de las personas que más amo. He descuidado la salud de mi familia.»

E6.03: «Durante los estudios de doctorado, ya no tenía tiempo libre para disfrutar de mis amigos y de las cosas que me gusta hacer con las personas que son parte de mi vida. Me encanta cantar y bailar.»

E10.06: «Yo decidí asumir el reto de la formación doctoral en el extranjero porque en Ecuador no se ofrece ese nivel de formación.»

Por otro lado, el reconocimiento de la comunidad respecto a la labor de los profesores es quizás otro de los elementos que motivado la transformación formativa de los participantes. Se extrae:

E1.04: «La adopción de estándares internacionales ha sido favorable para aportar al mejoramiento de las clases de Educación Física, deportes y recreación a mi retorno a Ecuador.»

E1.05: «Yo he acogido la investigación como eje principal para la formación de los estudiantes.»

E1.06: "La exigencia del grado de doctor para ejercer la docencia y la investigación en las universidades de Manabí, ha sido un proceso exigente, pero muy pertinente para avanzar en la gestión del conocimiento.»

E9:07: «A pesar de tener mis hijos ya adultos, el realizar el doctorado, me ha costado... por cuanto me ha obligado a alejarme mucho de mi familia para dedicarme al estudio.»

E10.08: «Ha sido una experiencia muy enriquecedora, se aprende mucho de la mano de mis tutores. Siempre he recibido apoyo los profesionales. [...] se avanza mucho y eso me tiene muy motivada para mejorar mi capacidad investigativa.»

A este punto, se infiere que el proceso de formación doctoral ha representado a los profesores distanciamiento de sus familias, parejas, vida social, entre otros. Mudarse fuera del país por periodos que oscilan entre 1 y 3 años ha generado el sentido de culpa. No obstante, se valora la alta contribución que representa el rol investigador para la solución de problemáticas locales que han persistido en el territorio.

Cambios en la identidad del profesorado y su rol investigativo 
A continuación, se presenta las afirmaciones recogidas en los grupos focales en los que los participantes abordaron los aspectos: (1) Promoción de la ciencia en la formación de profesionales del siglo XXI; (2) Participación de la comunidad científica global; y (3) Gestor de la ciencia para la superación de los problemas locales. Véase tabla 2.

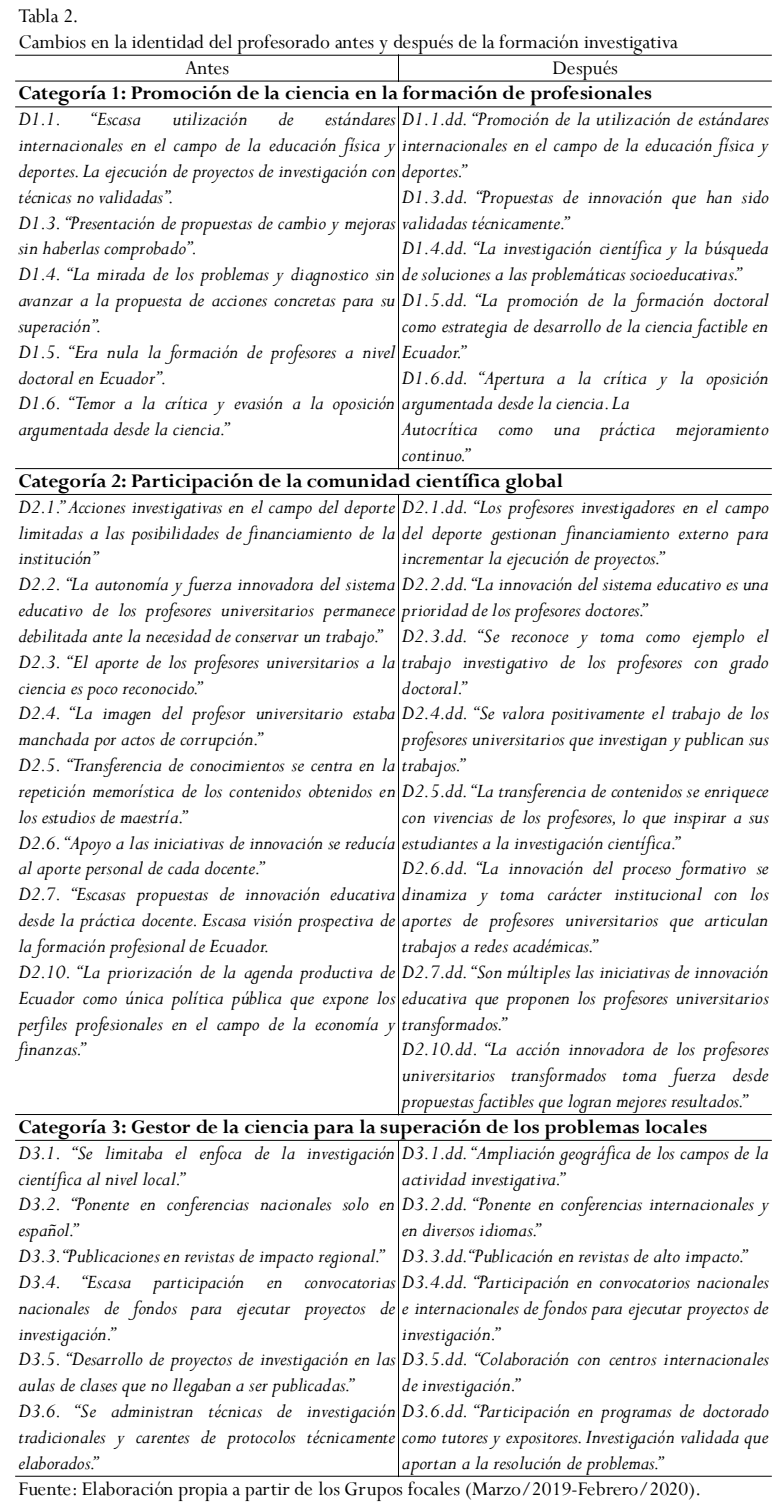

Cambios en la identidad de los participantes antes y después de la formación doctoral.

La transformación de la identidad del profesorado se vuelve notoria tras la formación doctoral y el retorno a las aulas en sus sitios de origen. En ese sentido, el extenso tiempo dedicado al autoaprendizaje, la formulación, ejecución de proyectos de investigación y a la producción científica son parte de sus rutinas. Los estilos de comunicación horizontal y empático con el alumnado; y la estimulación hacia el alumnado para el trabajo desde la ciencia son quizás las manifestaciones más claras de la transformación de la identidad.

\section{Percepciones de los participantes sobre su iden- tidad}

Se presenta las respuestas que los participantes dieron al cuestionario Construcción de la identidad de profesionales de la salud de (Jara y Mayor-Ruiz, 2018).Véase tabla 3.

\section{Tabla 3.}

Percepciones de los profesores respecto a las dimensiones de su identidad (N $=35)$

\begin{tabular}{|c|c|c|c|c|c|}
\hline \multirow{2}{*}{ 1. Dimensión Formación } & \multirow[t]{2}{*}{ MA } & \multirow{2}{*}{\multicolumn{2}{|c|}{ DA Ni DA, ni D }} & \multirow[t]{2}{*}{$\mathrm{D}$} & \multirow[t]{2}{*}{$\mathrm{MD}$} \\
\hline & & & & & \\
\hline 1 Facilitador aprendizaje & $68 \%$ & $28 \%$ & $1 \%$ & $3 \%$ & $0 \%$ \\
\hline 2 Reflexión del proceso & $26 \%$ & $8 \%$ & $11 \%$ & $43 \%$ & $12 \%$ \\
\hline 3 Motivador & $84 \%$ & $14 \%$ & $2 \%$ & $0 \%$ & $0 \%$ \\
\hline 4 Eficaz & $23 \%$ & $46 \%$ & $12 \%$ & $11 \%$ & $8 \%$ \\
\hline 5 Innovador & $18 \%$ & $58 \%$ & $8 \%$ & $16 \%$ & $0 \%$ \\
\hline 6 Dedicación a la investigación & $31 \%$ & $50 \%$ & $19 \%$ & $0 \%$ & $0 \%$ \\
\hline \multicolumn{6}{|l|}{7 Me encuentro desarrollado personal y } \\
\hline profesionalmente como docente & $28 \%$ & $69 \%$ & $3 \%$ & $0 \%$ & $0 \%$ \\
\hline 8 Falta capacitación en docencia & $32 \%$ & $41 \%$ & $8 \%$ & $11 \%$ & $9 \%$ \\
\hline 9 Falta desarrollo en investigación & $53 \%$ & $44 \%$ & $3 \%$ & $0 \%$ & $0 \%$ \\
\hline 10 Siento apoyo de las autoridades & $4 \%$ & $46 \%$ & $21 \%$ & $26 \%$ & $3 \%$ \\
\hline 11 Todavía desarrollándome & $34 \%$ & $63 \%$ & $0 \%$ & $3 \%$ & $0 \%$ \\
\hline $12 \mathrm{Me}$ gusta compartir experiencias & $39 \%$ & $43 \%$ & $7 \%$ & $9 \%$ & $2 \%$ \\
\hline \multicolumn{6}{|l|}{ 2. Dimensión Reflexión del proceso } \\
\hline 13 Función principal la docencia & $19 \%$ & $28 \%$ & $33 \%$ & $11 \%$ & $9 \%$ \\
\hline 14 Gestión-Docencia e investigación & $23 \%$ & $43 \%$ & $23 \%$ & $11 \%$ & $0 \%$ \\
\hline 15 Investigación y gestión & $56 \%$ & $36 \%$ & $8 \%$ & $21 \%$ & $4 \%$ \\
\hline 16 Falta tiempo protegido para investigar & $53 \%$ & $44 \%$ & $3 \%$ & $0 \%$ & $0 \%$ \\
\hline \multicolumn{6}{|l|}{ 3. Dimensión desempeño profesional } \\
\hline 17 Docencia-Investigación y Gestión & $12 \%$ & $47 \%$ & $29 \%$ & $8 \%$ & $4 \%$ \\
\hline 18 Docencia-Gestión e Investigación & $9 \%$ & $23 \%$ & $19 \%$ & $38 \%$ & $11 \%$ \\
\hline 19 Iguales proporciones & $14 \%$ & $33 \%$ & $28 \%$ & $21 \%$ & $4 \%$ \\
\hline 20 Muy difícil distribuir el tiempo & $49 \%$ & $12 \%$ & $14 \%$ & $14 \%$ & $11 \%$ \\
\hline 21 De acuerdo con los cargos de gestión que ocupa & $21 \%$ & $29 \%$ & $20 \%$ & $7 \%$ & $23 \%$ \\
\hline 22 Docencia y Gestión & $47 \%$ & $34 \%$ & $19 \%$ & $0 \%$ & $0 \%$ \\
\hline 23 Falta estabilidad laboral & $22 \%$ & $28 \%$ & $13 \%$ & $20 \%$ & $17 \%$ \\
\hline \multicolumn{6}{|l|}{24 La motivación para la docencia se perjudica por el } \\
\hline clima laboral & $38 \%$ & $35 \%$ & $8 \%$ & $19 \%$ & $0 \%$ \\
\hline 25 Faltan recursos para docencia e investigación & $53 \%$ & $44 \%$ & $3 \%$ & $0 \%$ & $0 \%$ \\
\hline 26 Desarrollo en docencia y gestión & $24 \%$ & $43 \%$ & $11 \%$ & $14 \%$ & $8 \%$ \\
\hline 27 Falta capacitación en gestión & $15 \%$ & $21 \%$ & $4 \%$ & $43 \%$ & $17 \%$ \\
\hline \multicolumn{6}{|l|}{$\begin{array}{l}\text { 4. Dimensión Elementos afectivos de la } \\
\text { identidad }\end{array}$} \\
\hline 28 Compromiso & $67 \%$ & $22 \%$ & $9 \%$ & $2 \%$ & $0 \%$ \\
\hline 29 Responsabilidad & $63 \%$ & $32 \%$ & $4 \%$ & $1 \%$ & $0 \%$ \\
\hline 30 Cercano al estudiante & $24 \%$ & $37 \%$ & $7 \%$ & $32 \%$ & $0 \%$ \\
\hline 31 Dominio del tema & $85 \%$ & $15 \%$ & $0 \%$ & $0 \%$ & $0 \%$ \\
\hline 32 Proactivo & $26 \%$ & $48 \%$ & $8 \%$ & $12 \%$ & $6 \%$ \\
\hline 33 Exigentes & $42 \%$ & $43 \%$ & $15 \%$ & $0 \%$ & $0 \%$ \\
\hline 34 Empatía & $35 \%$ & $56 \%$ & $4 \%$ & $5 \%$ & $0 \%$ \\
\hline
\end{tabular}

Percepciones del profesorado sobre las categorías de su identidad

Para la dimensión formación de la identidad del profesorado de Educación física y deportes; Finanzas y economía; y Psicología educativa, los ítems que alcanzaron mayor frecuencia son: 4. Motivador y 2. Facilitador aprendizaje. Llama la atención que el ítem 3. Reflexión del proceso, alcanza una alta frecuencia en desacuerdo lo que sugiere que los profesores pocas veces analizan su desempeño en la enseñanza.

Para la dimensión Reflexión del proceso se indica que el ítem que reporta mayor frecuencia es el 17. Se necesita tiempo protegido para investigar. Esta respuesta sugiere que el profesorado distribuye su tiempo entre diversas actividades, limitando su dedicación a la investigación. Esto se ratifica en la respuesta al ítem 13. Función principal la docencia; donde la mayor frecuencia está en la respuesta imparcial. 
Para la dimensión desempeño profesional los ítems con mayor frecuencia son el 25. Faltan recursos para docencia e investigación; y el número 20. Muy difícil distribuir el tiempo. El ítem que reporta la mayor frecuencia en desde el desacuerdo es el 20. Falta capacitación en gestión, de lo que se infiere que el profesorado ya ha sido capacitado en este aspecto. Llama la atención el ítem 23. Falta estabilidad laboral, ya que es una minoría el profesorado que se mantienen en estatus laboral de contratación ocasional.

Para la dimensión Elementos Afectivos de la identidad. En este grupo aparece el ítem que alcanzó la más alta frecuencia de todo el instrumento. 31. Dominio del tema, seguido por 28. Compromiso y el ítem 29. Responsabilidad.

Comparación de los ítems relacionados al rol investigativo en la identidad del profesorado de Educación Física y Psicología Educativa

En la tabla 4 se presenta la frecuencia reportada por el profesorado de Educación Física y Psicología Educativa respecto a los ítems relacionados al rol investigativo.

Respecto al ítem 6. Dedicación a la investigación, tanto la mayoría del profesorado de Educación Física como el de Psicología Educativa indican una tendencia de dedicación medía a procesos de investigación científica; $28,60 \%$ y $22,90 \%$ respectivamente.

En cuanto al ítem 9. Falta desarrollo en investigación, el 31,43\% el profesorado de Educación Física expresa una tendencia alta; mientras que el de Psicología Educativa indica una tendencia moderada 25,70\%.

Para el ítem 15. Investigación y gestión, el 25,70\% del profesorado de Educación Física expresa una tendencia moderada; mientras que el 34,29\% el de Psicología Educativa indica una tendencia alta.

En cuanto al ítem 16. Falta tiempo para investigación, el $34,29 \%$ del profesorado de Educación física indica su mayor frecuencia en el nivel alto; mientras que el de

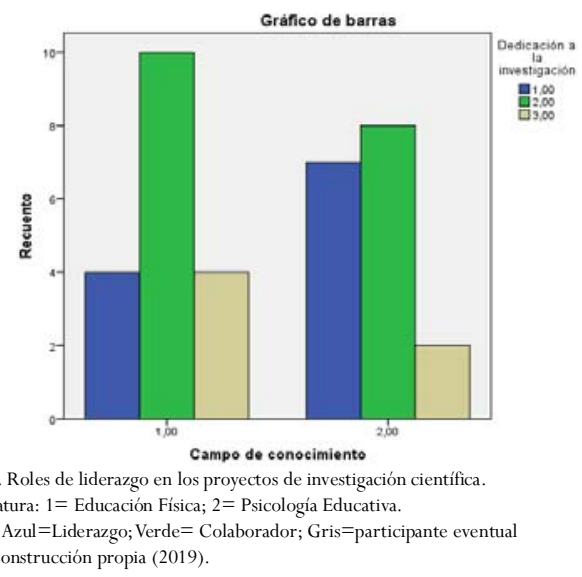

Psicología Educativa se concentra su máxima votación en nivel medio con 28,60\%.

Finalmente, todo el profesorado coincide respecto al ítem 26. Faltan recursos para la investigación. Ellos expresan una alta tendencia con $28,57 \%$ para el profesorado de Educación Física y 25,71\% para el de Psicología Educativa.

En el gráfico 1 se expone la frecuencia con la que el profesorado de Educación Física lidera los procesos investigativos es menor en comparación el de Psicología Educativa.

Sin embargo, la frecuencia con la que el profesorado de Educación Física asume los roles de colaboradores operativos (barra verde) y apoyos eventuales (barra gris) en procesos investigativos es mayor en comparación del profesorado de Psicología Educativa.

\section{Relación entre campos del conocimiento y rol investigativo}

$\mathrm{La} \mathrm{Ho}_{\mathrm{o}}=$ No existen relaciones significativas entre los campos del conocimiento del profesorado y el rol investigativo.

Al ejecutar la prueba Kolmogorov-Smirnov se determina que las series de datos no son normales. Por lo tanto, se aplica la prueba no paramétrica U de MannWhitney con un nivel de significación $p$-valor $=.050$; Véase tabla 4.

\begin{tabular}{|c|c|c|c|c|c|c|c|c|c|}
\hline Participantes & Alto\% & & Medio\% & & Bajo & & & Total & $p$-valor $=.050$ \\
\hline \multicolumn{10}{|c|}{ 6. Dedicación a la investigación } \\
\hline Educación Física & & $11,43 \%$ & 10 & $28,60 \%$ & 4 & $11,00 \%$ & 18 & $51,43 \%$ & .258 \\
\hline Psicología Educativa & & $20,00 \%$ & 8 & $22,90 \%$ & 2 & $5,70 \%$ & 17 & $48,57 \%$ & \\
\hline \multicolumn{10}{|c|}{ 9. Falta desarrollo en investigación } \\
\hline Educación Física & 11 & $31,43 \%$ & 7 & $20,00 \%$ & 0 & $0,00 \%$ & 18 & $51,43 \%$ & .483 \\
\hline Psicología Educativa & 8 & $22,86 \%$ & 9 & $25,70 \%$ & 0 & $0,00 \%$ & 17 & $48,57 \%$ & \\
\hline \multicolumn{10}{|l|}{ 15. Investigación y gestión } \\
\hline Educación Física & 8 & $22,86 \%$ & 9 & $25,70 \%$ & 1 & $2,90 \%$ & 18 & $51,43 \%$ & .219 \\
\hline Psicología Educativa & 12 & $34,29 \%$ & 4 & $11,40 \%$ & 1 & $2,90 \%$ & 17 & $48,57 \%$ & \\
\hline \multicolumn{10}{|c|}{ 16. Falta tiempo para investigación } \\
\hline Educación Física & 12 & $34,29 \%$ & 4 & $11,40 \%$ & 2 & $5,70 \%$ & 18 & $51,43 \%$ & .351 \\
\hline Psicología Educativa & & $20,00 \%$ & 10 & $28,60 \%$ & 0 & $0,00 \%$ & 17 & $48,57 \%$ & \\
\hline \multicolumn{10}{|c|}{ 26. Faltan recursos para la investigación } \\
\hline Educación Física & 10 & 28,57 & 8 & $22,90 \%$ & 0 & $0,00 \%$ & 18 & $51,43 \%$ & .901 \\
\hline Psicología Educativa & 9 & 25,71 & 8 & $22,90 \%$ & 0 & $0,00 \%$ & 17 & $48,57 \%$ & \\
\hline
\end{tabular}

Todos los resultados de la prueba no paramétrica U de Mann. Whitney entre los factores del rol investigativo superan $p$-valor $=.050$ tanto para el profesorado de Educación física como para el profesorado de Psicología Educativa. Por lo tanto, se acepta la hipótesis nula para todos los casos. Así, se demuestra que no existen relaciones significativas entre el profesorado de Educación Física y Psicología Educativa y los factores del rol investigador.

Los resultados del presente trabajo confirman las 
afirmaciones de Alonso et al (2015) y Moya y Alonso (2016) respecto a que el profesorado integra los recursos didácticos, pedagógicos, materiales y humanos para proponer y poner en marcha innovaciones al proceso formativo de los profesionales. Sin embargo, en este estudio el grupo de profesores participantes se convierte en promotores del uso de la ciencia en el contexto local y nacional, superando las fronteras de la formación profesional para asumir el rol investigador del origen subyacente de las problemáticas socioeducativas. Así, ellos han logrado contribuir al mejoramiento de la producción científica de Ecuador y han llevado a sus alumnos a nuevos y mejores niveles de aplicación de la ciencia, lo que encuentra parcial armonía con las afirmaciones de Rojas (2016) respecto al aprender a aprender, a hacer y a ser. Sin embargo, los autores de este trabajo encuentran mayor armonía de los resultados obtenidos con las afirmaciones de Keiler (2018), cuando insiste en la necesidad de avanzar hacia la educación del siglo XXI, la que requieren de habilidades como la creatividad y la innovación, el pensamiento crítico y la resolución de problemas, la comunicación y la colaboración.

De la misma forma, a la luz de los datos se ratifica la posición de Gajardo-Asbún (2019), que la identidad del profesorado universitario es un proceso en evolución permanente. Este inicia como un proceso individual que se consolida en el espacio universitario donde las transformaciones reciben el apoyo o la oposición en procura del fortalecimiento de la academia de Ecuador. Así, la política pública posibilitó la movilidad del profesorado para cursar la formación doctoral en el extranjero mediante diversas modalidades de estudio, pero su dedicación y priorización demandó de sacrificios personales, familiares, económicos, etc., que complementaron la transformación de la identidad del profesorado que asumieron los retos del perfeccionamiento en el campo investigativo, motivados por el deseo de aportar al desarrollo de sus comunidades. Por lo tanto, las transformaciones de los participantes trascienden la individualidad para convertirse en un aporte colectivo que suma al desarrollo de la ciencia en la región.

A la luz de los resultados se ratifican las afirmaciones de Nghia \& Tai (2017) respecto a los significados y motivaciones de las personas para optar por la profesión de educador. Sin embargo, este estudio también aborda los esfuerzos y sacrificios hechos por los participantes para lograr la meta de la formación doctoral, la que inicia por el cumplimiento de la política pública, pero que en lo posterior se convierte en el deseo legítimo de contribuir desde el uso de la ciencia, a la superación de problemáticas educativas que persisten.

En este trabajo se remarca que, desde la movilidad del profesorado emergen procesos de mayor interacción entre los colegas de diversas naciones y campos del conocimiento, lo que fortalece el rol investigador, y trasciende al alumnado, a quienes contribuye en la comprensión profunda del contenido y el desarrollo de las habilidades de ajuste a los cambios permanentes (Keiler, 2018).

Las voces de los participantes encuentran sintonía con las afirmaciones de Padilla et al. (2015) y García et al. (2018) respecto a que las universidades requieren de profesorado altamente capacitado para contribuir al mejoramiento de la calidad de la educación.

El profesorado ha dinamizado los procesos formativos y sus frutos han aportado a la superación de problemáticas socioeducativas de Ecuador, resultado que es coherente con la posición de Sierra (2015) respecto al ideal de que cada educador responda a las necesidades del contexto donde ejecuta su trabajo. Así, el fortalecimiento del rol investigativo ha permitido acercar el alumnado a los espacios de encuentro científico, el uso de instrumentos utilizados en procesos de investigación rigurosos, y el cultivo de valores y visión crítica ante la vida, la cultura y el ambiente.

De la triangulación de la información obtenida mediante las entrevistas y los grupos focales se infiere: (a) los participantes han realizado diversa aportes en la innovación de los procesos formativos que estimulan en el alumnado, el aprendizaje autónomo, el enfoque de la investigación científica ante los problemas locales, y la práctica del pensamiento crítico, (b) el profesorado enfrenta dificultades para lograr el balance entre las funciones de la docencia, la investigación y la vinculación con la comunidad y la conciliación con los aspectos personales y familiares. Por lo tanto, asumir la función investigativa también puede causar desequilibrio en la vida personal del profesorado, tal como lo afirman $\mathrm{Ca}-$ ballero \& Bolívar (2015) y Granados et al. (2017) respecto a que, el incremento de la dedicación a la investigación científica resta la dedicación a tareas de planificación, ejecución y seguimiento del proceso de enseñanza, (c) las políticas públicas para el aseguramiento de la calidad de la educación superior plantean el crecimiento de todas las funciones sustantivas, acorde con los estándares del modelo de evaluación de la calidad de la educación superior, y (d) los participantes trabajan en el rescate de la cultura propia y la apertura al conocimiento de otras, la aceptación de diferencias, la tolerancia a la oposición y la concentración en la búsqueda 
de soluciones a problemáticas que persisten en el territorio tales como la sobre explotación de los recursos naturales, violencia social e inequidad de género, y la atención a los grupos vulnerables de la sociedad.

Los resultados obtenidos mediante la triangulación de la información encuentran coincidencia con las afirmaciones de Polaino \& Romillo (2017) y Saltos (2018) respecto a la necesidad de que se reafirme la responsabilidad social de las universidades en Ecuador.

En cuanto al estudio de las relaciones existentes entre los ítems del Cuestionario para la Construcción de la identidad del profesional de Jara \& Mayor-Ruiz (2018) se indica que, una vez ajustado el instrumento al contexto del profesorado universitario de Ecuador, se detectó que no existen relaciones significativas entre los ítems del rol investigativo con los factores sexo, situación laboral y campo del conocimiento, excepto para el ítem 15. Investigación y gestión, en el que se encontró relación significativa con el factor -Situación laboral-.

La triangulación de los resultados del Cuestionario para la Construcción de la Identidad del Profesional con la información generada mediante las entrevistas y los grupos focales permite inferir que, (1) las transformaciones de la identidad del profesorado responden positivamente a las expectativas de la comunidad universitaria, (2) el profesorado transformado asume el rol de actor social, crítico y promotor del desarrollo socioeducativo de Ecuador.

Las nuevas identidades del profesorado de Educación Física y Psicodidáctica se evidencian en su desempeño dentro y fuera de las aulas (Uitto et al., 2015 y Alonso et al., 2015). Así, los aportes de esta investigación pueden contribuir al mejoramiento de la política pública para la educación superior y la revisión de las políticas institucionales de las universidades y Escuelas Politécnicas de Ecuador.

Entre los hallazgos no previstos en el marco de esta investigación, se indica que el tiempo que los informantes se han mantenido distantes del hogar y la convivencia en contextos educativos en el extranjero han influido en el cambio de los estilos de comunicación hacia el alumnado, y se detecta mayor apertura y empatía ante las dificultades que enfrentan las autoridades universitarias y los colegas. De esta manera, se ratifica que el encargo social hecho a las universidades que exponen Morales et al. (2019) y Rueda et al. (2020) toma mayor fuerza cuando se cuenta con que el profesorado posee una visión global, hace uso de estilos de comunicación horizontal y promueven la práctica investigativa como generador de nuevo conocimiento y su transferencia.

\section{Conclusión}

A partir de la revisión teórica y los resultados del proceso empírico, se declara el cumplimiento del objetivo propuesto para este estudio. Así, se concluye que al fortalecerse el rol investigativo en la identidad del profesorado se potencia el cumplimiento de las políticas públicas de la educación superior de Ecuador. Los análisis estadísticos para la comprobación de la hipótesis muestran que no existen diferencias significativas entre el profesorado de Educación Física y Psicodidáctica respecto al rol investigativo. Por lo tanto, todos los participantes han fortalecido el rol investigador sin diferencias por el campo de trabajo que tienen.

La contribución principal de este trabajo se centra en la determinación de los cambios del rol investigativo, el aporte a la innovación de los procesos de formación de profesionales, y la visión crítica y proactiva para la superación de las problemáticas que persisten en el contexto.

Los resultados presentados pueden ser utilizados en la toma de decisiones institucionales para la construcción de proyectos diversos relacionados con la identidad del profesorado, y a nivel de la política pública este trabajo visibiliza resultados de la inversión nacional realizada para el perfeccionamiento del profesorado entre 2010-2020. Sin embargo, la oferta de programas de formación doctoral en Ecuador sigue siendo limitada, lo que sugiere a la comunidad científica trabajar en ese horizonte. Aunque, las recientes políticas de gobierno han priorizado la oferta de programas de formación técnica como ruta para el acceso al mercado laboral.

La debilidad de este trabajo es quizás el tamaño del corpus que se concentra en dos de las 24 provincias de Ecuador. Sin embargo, el estudio propone una ruta para que se repliquen nuevos trabajos en esta línea.

A manera de prospectiva respecto al nuevo perfil del profesorado se añade que el alumnado encuentra en ellos los nuevos modelos de profesionales y promotores de procesos para la construcción del conocimiento científico y en la educación del siglo XXI.

\section{Agradecimientos}

Este trabajo es parte de una investigación más amplia ejecutada en el marco del Programa de Doctorado en Psicodidáctica y Didácticas Específicas de la Universidad del País Vasco, España.

Este trabajo además se suscribe a los Proyectos de la Facultad de Ciencias de la Educación de la Universidad 
Laica Eloy Alfaro de Manabí: (1) Comprensión Lectora y Escritura Académica, (2) Innovación y desarrollo de procesos educativos; y la Red de investigación socioeducativa LEA: Cambiando vidas, en el que colaboran docentes de la Pontificia Universidad Católica de Ecuador Sede Esmeraldas y Universidad Laica Eloy Alfaro de Manabí.

\section{Referencias}

Alvarado, L. y García, M. (2008). Características más relevantes del paradigma socio-crítico: su aplicación en investigaciones de educación ambiental y de enseñanza de las ciencias realizadas en el Doctorado de Educación del Instituto Pedagógico de Caracas Sapiens. Revista Universitaria de Investigación, 9(2), 187-202.

Alonso, I., Lobato, C., \& Arandia, M. (2015). La identidad profesional docente como clave para el cambio en la educación superior. Opción, 31(5), 51-74. Recuperado de https://www.redalyc.org/pdf/310/ 31045570004.pdf

Anversa, A. L., Souza, V. de F., Both, J., da Costa, L. C., Batista, P., \& Oliveira, A. (2020). Escala de avaliação da constituição da identidade profissional do bacharel em educação física. Retos, 38(1) 196-203. https: / / doi.org/10.47197/retos.v38i38.74749

Antuña, S. (2014). La investigación no es para los demás. Revista Española de Cirugía Ortopédica y Traumatología, 58(3), 129-130. doi:10.1016/ j.recot.2014.02.004

Bell, R. (2017). Identidad profesional y componentes de las competencias profesionales del docente universitario en el siglo XXI. Yachana-revista cientifica, 6(2), 94-105. Recuperado de http: / / revistas.ulvr.edu.ec/ index.php/yachana/article/view/478/267

Bermejo, J. M., Pulido, D., Galmés, A., Serra, P., Vidal, J., \& Ponseti, F. J. (2020). Educación física y universidad: Evaluación de una experiencia docente a través del aprendizaje cooperativo. Retos, 39(1), 9097. https: / / doi.org/10.47197/retos.v0i39.77834

Bernate, J. A., Guataquira Romero, A., Romero-Melo, E. N., \& Reyes Escobar, P. C. (2020). Satisfacción de la Calidad Educativa en Educación Superior. PODIUM, 38(1), 37-50. https://doi.org/10.31095/ podium.2020.38.3

Caballero, K. y Bolívar, A. (2015). El profesorado universitario como docente: Hacia una identidad profesional que integre a la docencia e investigación. REDU, 13(1), 57-77. Recuperado de http://redu.net/redu/files/journals/1/articles/900/public/
900-3924-1-PB.pdf

Cevallos, D. (2018). las universidades intervenidas por el consejo de educación superior en Ecuador. (Tesis de Doctorado). Universidad de Barcelona. Programa de Doctorado Educación y Sociedad.

Cuevas, A. (2016). La educación superior ante los desafíos sociales. Alteridad, 11(1), 101-109. Recuperado de https://www.redalyc.org/pdf/4677/ 467746763008.pdf

Darley, W. y Luethge, D. (2016). The Role of Faculty Research in the Development of a Management Research and Knowledge Culture in African Educational Institutions. Academy of Management Learning \& Education, 15(2), 1-18. DOI: https: / / doi.org/10.5465/amle.2013.0337

Difabio de Anglat, H., Portela de Nieto, A., GelonchVillarino, S., Muscará, F., \& Boarini de Dutto, M. (2018). La experiencia de investigación educativa de nivel doctoral en la región de Cuyo, Argentina. REDU, 16(1), 11-32.DOI: https: / /doi.org/10.4995/ redu.2018.5690

Domínguez-Lara, S. A. y Merino-Soto, C. (2015). ¿ Por qué es importante reportar los intervalos de confianza del coeficiente alfa de Cronbach?. Revista Latinoamericana de Ciencias Sociales, Niñez y Juventud, 13(2), 1326-1328. Recuperado de http:// revistaumanizales.cinde.org.co/rlcsnj/index.php/ Revista-Latinoamericana/article/view/2030

Edwards, F. C. y Edwards, R. J. (2016). A story of culture and teaching: the complexity of teacher identity formation. Curriculum Journal, 28(2), 190-211. Recuperado de http: https: / /www.tandfonline.com/

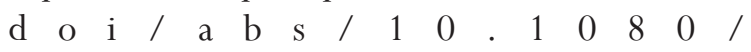
09585176.2016.1232200?journalCode $=$ rcjo20

Fastuca, L. F. y Wainerman, C. (2015). La dirección de tesis de doctorado: ¿una práctica pedagógica? Perfiles Educativos, 37(148), 156-171. doi:10.1016/ j.pe.2015.11.013

Gajardo-Asbún, K.P. (2019). Estado del arte sobre identidad docente: Investigación de experiencias de profesores en formación y en ejercicio. Rev. investig. educ. REDIECH, 10(18), 1-20. Recuperado de http:// w w w. s c i e l o . o r g. $\mathrm{m}$ x / scielo.php?script $=$ sci_arttext\&pid $=$ S 2448 85502019000100079.

García, W., Jara, G., \& Cedillo, M. (2018). Experiencias sobre la articulación de las funciones sustantivas en Educación Superior. Revista Killkana Sociales, 2(3), 115-120. DOI: https://doi.org/10.26871/ killkana_social.v2i3.334 
Granados, J., Tapia, A., \& Fernández, J. (2017). La construcción de la identidad de los docentes noveles: un análisis desde las teorías apriorísticas. REDU, 15(2), 163-178. Recuperado de https:/ / polipapers.upv.es/ index.php/REDU/article/view/6746/9202

Guerreo-Aray, G. R., Suástegui-Solórzano, S., \& Zambrano-Vera, G.E. (2019). La calidad de la enseñanza en la Educación Superior en Ecuador. Dominios de la Ciencia, 5(3), 258-270. DOI:10.23857/ dc.v5i3.934

Gutiérrez, M. G. (2013). Innovar o morir: perspectivas desde el ámbito educativo. RETOS. Revista de Ciencias de la Administración y Economía, 3(6), 139-150. Recuperado de https:/ / retos.ups.edu.ec/index.php/retos/article/view/6.2013.02

Guzmán, L. (2017). Construcción de la identidad profesional docente: Estudio cualitativo sobre la construcción de la identidad profesional de los estudiantes de pedagogía en programas de formación inicial de profesores de carácter público y privado. Tesis doctoral Universidad de Girona. Recuperado de https:// dialnet.unirioja.es $/$ servlet $/$ tesis?codigo $=158326$

Guzmán, J. y Martínez, M. (2016). Tensiones en la construcción de identidades académicas. Estudios Pedagógicos, XLII(3), 191-206. Recuperado de https:// scielo.conicyt.cl/pdf/estped/v42n3/art10.pdf

Ismail, R. y Meerah, T. S. M. (2012). Evaluating the Research Competencies of Doctoral Students. Procedia - Social and Behavioral Sciences, 59(1), 244 247. doi:10.1016/j.sbspro.2012.09.271

Jara, C. y Mayor-Ruíz, C. (2018). Explorar la Construcción de la Identidad Docente en Profesionales de la Salud: Diseño y Validación de Instrumento. Formación Universiaria, 12(1), 13-24. Recuperado de https://scielo.conicyt.cl/pdf/formuniv/v12n1/ 0718-5006-formuniv-12-01-00013.pdf

Jimenez, M. (2019). Identidad académica: una franquicia en construcción. Revista Educar, 55(2), 543-560. Recuperado de https://www.raco.cat/index.php/ Educar/article/view/v55-n2-jimenez/451334

Keiler, L.S. (2018). Teachers' roles and identities in student-centered classrooms. IJ STEM Ed 5(34), 120. DOI: https://doi.org/10.1186/s40594-0180131-6

Maldonado, C. I. y Ávila, W. L. (2014). Evaluación del desempeño del egresado de la carrera de Ingeniería Comercial de la Universidad Politécnica SalesianaEcuador. Retos, 4(7), 45-61. Disponible en https:// retos.ups.edu.ec/index.php/retos/article/view/ 7.2014 .03
Morales, C. y Rueda, D. (2019). Aproximaciones teóricas a la calidad de la docencia universitaria. Revista Cubana de Educación Superior, 38(2). Recuperado de h t t p : / / s c i e l o.s l d . c u / scielo.php?script $=$ sci_arttext\&pid $=$ S025743142019000200002

Morales-Román, G. (2015). Retrato narrativo de las experiencias, retos y motivaciones de la mujer adulta: estudiante del Programa Doctoral en Educación. Revista Iberoamericana de Educación Superior, 6(16), 143163. doi:10.1016/j.rides.2014.05.001

Moreira, T.V., Fleitas, S., Veliz, V. \& Vinces, C. J. (2016). La vinculación con la sociedad en los patrones de calidad de universidades ecuatorianas e hispanoamericanas Dilemas Contemporáneos. Educación Politicas y Valores, 4(1), 1-25.

Moscoso, J. N. (2017). Los métodos mixtos en la investigación en educación: hacia un uso reflexivo. Cadernos de Pesquisa, 47(164), 632-649. Recuperado de https:/ /www.scielo.br/scielo.php?pid=S0100 $15742017000200011 \&$ script $=$ sci_arttext

Moya, C. y Alonso, L. (2016). Modelo de formación de la competencia en marketing para estudiantes de la carrera de economía formación de la competencia en marketing para estudiantes de economía. Revista REFCalE, 4(1), 85-105. Recuperado de http:// www.refcale.uleam.edu.ec/index.php/refcale/ article/view/1102/669

Nieva, J. y Mendoza, K. (2019). Estrategia pedagógica de formación docente desde una perspectiva desarrolladora. RECUS, 4(3), 05-09. Recuperado de https://revistas.utm.edu.ec/index.php/Recus/ article/view/2170/2350

Nghia, L. H. y Tai, N. (2017). Preservice Teachers' Identity Development during the Teaching Internship. Australian Journal of Teacher Education, 42(428), 1-20. Recuperado de https:// ro.ecu.edu.au/ajte/vol42/iss8/1/

Núñez, M. N. I. y Santesteban, E. O. (2017). Cómo iniciarse en investigación durante la residencia: recomendaciones para la elección de tema de investigación, universidad y mentor. Psiquiatría Biológica, 24(3), 118-124. doi:10.1016/j.psiq.2017.10.001 .

Orozco-Inca. E. E., Jaya-Escobar, A. I., Ramos-Azcuy, F. J., \& Guerra-Bretaña. R. M. (2020). Retos a la gestión de la calidad en las instituciones de educación superior en Ecuador. Educación Médica Superior, 34 (2), 1-14.

Pacheco-Méndez, T. (2014). Tradición, contexto y objeto de estudio en las tesis doctorales en Educación de 
tres universidades. Revista Iberoamericana de Educación Superior, 5(12), 46-69. doi:10.1016/s 2007 2872(14)71942-0

Padilla, A., López, M., \& Rodríguez, A. (2015). La formación del docente universitario. Concepciones teóricas y metodológicas. Revista Universidad y Sociedad, 7(2). 86-90. Recuperado de http://scielo.sld.cu/ pdf/rus/v7n1/rus11115.pdf

Palamidessi, M. I., Gorostiaga, J. M., \& Suasnábar, C. (2014). El desarrollo de la investigación educativa y sus vinculaciones con el gobierno de la educación en América Latina. Perfiles Educativos, 36(143), 49-66. doi:10.1016/s0185-2698(14)70609-9

Polaino, C. y Romillo, A. (2017). Vinculación con la Sociedad en la Universidad de Otavalo,Ecuador. Formación universitaria, 10(3), 21-30. Recuperado de https://www.redalyc.org/pdf/3735/ 373551306004.pdf

Palomar, C. (2013). Culturas académicas Prácticas y procesos de formación para la investigación en doctorados en educación. Perfiles Educativos, 35(139), 204 212. Doi:10.1016/s0185-2698(13)71817-8

Ramiréz, R. (2016). Universidad Urgente para una sociedad emancipada. SENESCYT, Quito - Ecuador.

Ramírez, R., Cevallos Vallejos, M., Díaz Reinoso, X., Grijalva, A., Rojas, G., Rueda Novoa, R., \& Santos Jara, E. (2016). CES: cinco años construyendo una educación superior incluyente, de calidad y pertinente. (C. de educación Superior, Ed.). Quito, Ecuador.

Rivera, C., Espinosa, J., \& Valdés, Y. (2017). La investigación científica en las universidades ecuatorianas. Prioridad del sistema educativo vigente. Revista $\mathrm{Cu}$ bana Educación Superior, 36(2), 113-125. Recuperado d e h t t p : / / s c i e l o.s l d . c u / scielo.php?script $=$ sci_arttext $\&$ pid $=$ S02 57 43142017000200011

Rojas, M. (2016). Determinación del perfil de competenciasdel docente universitario, desde la mirada del académico, en el marco de un modelo orientado al desarrollo de competencias de los estudiantes en la Univesidad SantoTomas. Tesis Doctoral Chile. Recuperado de https: / / riuma.uma.es/xmlui / handle/10630/12192

Rueda, I., Acosta, B., \& Cueva, F. (2020). Las Universidades y sus prácticas de vinculación con la sociedad. Educ. Soc., Campinas, 41(1), 1-20. Recuperado de https: / /www.scielo.br/pdf/es/v41/1678-4626-es41-e218154.pdf

Saltos, G., Odriozola, S., \& Ortiz, M. (2018). La vinculación Universidad-Empresa -Gobierno: Una visión histórica y conceptual. Revista ECA Sinergia., 9(2), 121 139. Recuperado de https://revistas.utm.edu.ec/ index.php/ECASinergia/article/view/1466

Sáez, M. B. S., Gil, P. G., \& Martínez, M. M. (2021). Desarrollo psicomotor y su vinculación con la motivación hacia el aprendizaje y el rendimiento académico en Educación Infantil. Revista de educación, (392), 177-203. https: / / dialnet.unirioja.es/servlet/ articulo?codigo $=7826445$

Sierra, G. (2015). Modelo educativo. Formación en competencias de la Universidad EAN. Recuperado de línea. http:/ / institución.universidadean.edu.co/ documents/assessment-center/Evaluacion-dedesempeno-docentes.pdf.

Tapia,A., Granados, J., \& Fernández, J. (2017). La construcción de la identidad de los docentes noveles: un análisis desde las Teorías apriorísticas. Dominios de la Ciencai, 3(2), 40-65. Recuperado de https:// webcache.googleusercontent.com / search?q= cache:sEPy09fOxd4J:https: / / polipapers.upv.es/index.php/REDU/article/ download / 6746/9202+\&cd $=2 \&$ hl $=$ es 419\&ct $={ }_{\mathrm{clnk}} \& \mathrm{gl}={ }_{\mathrm{ec}}$

Uitto, M., Kaunisto, S. L., Syrjälä, L., \& Estola, E. (2015). Silenced Truths: Relational and Emotional Dimensions of a Beginning Teacher's Identity as Part of the Micropolitical Context of School. Scandinavian Journal of Educational Research, 59(2), 162-176. Recuperado de https: / / www.tandfonline.com/doi/abs/ 10.1080/00313831.2014.904414

Vallejo-López, A. B. (2020). The role of the university professor in the training of researcher students from the early stage. Educ Med Super, 34(2), 1-20.

Véliz Briones, V. F. (2018). Calidad en la Educación Superior. Caso Ecuador. Atenas, 1(41), 165-180.

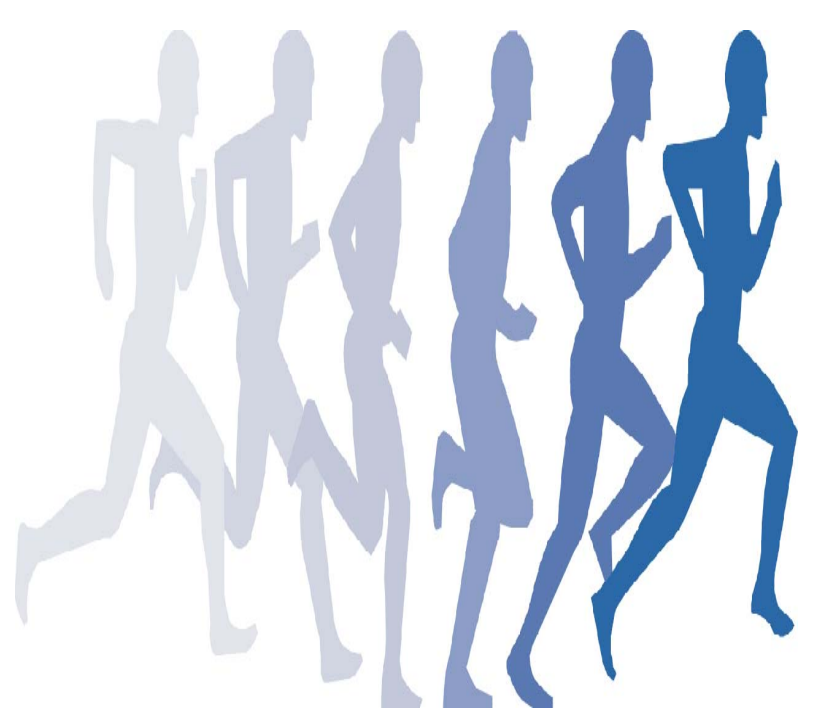

